\title{
Advances in Endoscopic Sinus Surgery Technique: The Impact of Technological Breakthroughs
}

\author{
NR London Jr' ${ }^{1}$, SJ Zinreich ${ }^{2}$ and FA Kuhn ${ }^{3 *}$ \\ ${ }^{1}$ Department of Otorhinolaryngology Head and Neck Surgery, The Johns Hopkins Medical Institutions, Baltimore, Maryland, USA \\ ${ }^{2}$ Russel H. Morgan Departments of Radiology, The Johns Hopkins Medical Institutions, Baltimore, Maryland, USA \\ ${ }^{3}$ Georgia Nasal \& Sinus Institute Savannah, GA, USA
}

*Corresponding author: FA Kuhn, Georgia Nasal \& Sinus Institute Savannah, GA, USA.

Received Date: December 12, 2020

Published Date: January 05, 2021

\section{Opinion}

Messerklinger initially characterized sinonasal mucociliary clearance patterns in the 1950's using India ink particles and hypothesized that inflammatory disease within the sinuses, as well as anatomic variants, could obstruct mucus outflow. He introduced use of the endoscope for diagnostic evaluation, as well as surgical principles known as the Messerklinger technique [1]. In the following 70 years his technique, known as Endoscopic Sinus Surgery (ESS), was refined and popularized by Stammberger, Kennedy, and others [2,3]. Both emphasized the preservation of normal anatomic structures and re-establishment of natural mucociliary clearance patterns. Towards that end, Kennedy viewed the surgery as a functional and minimally invasive procedure, adding the term "functional" to ESS, with the procedure now widely known as Functional Endoscopic Surgery (FESS) [4]. Since then, minimally invasive endoscopic techniques have been further refined by a multitude of talented multi-disciplinary physicians, too numerous to name here [5].

Technological imaging advances have paralleled these developments. X-ray polytomography was replaced by multiplanar computed tomography (CT) as functional sinus surgery was in its infancy [6]. In addition to opening and enlightening our view of paranasal sinus anatomy, multiplanar CT imaging gave rise to intraoperative image guidance opportunities. More recently, threedimensional computer x-ray tomography stereoscopic imaging (3DCTSI) has been developed, which allows the viewer to evaluate the CT image with volume rendering and depth perception [7].
Augmented and virtual reality systems have also shown promise in aiding intraoperative navigation and as educational tools [8]. The impact of these technological advances on the understanding of newly identified anatomic outflow relationships, however, has not been fully understood or applied to endoscopic sinus surgery.

A recent study by Zinreich, et al. [6] has demonstrated new understanding of anatomic relationships that can only be appreciated, when using 3DCTSI techniques to study sinonasal anatomy [4]. In this study of the frontal sinus drainage pathway, new concepts regarding spaces, uncinate process segments, their attachment sites and the resulting frontal sinus outflow pathway were carefully described. The technological advances were critical to make these observations feasible. Virtual/augmented reality and 3DCTSI technology also allow for 'virtual' surgery in which obstructing anatomic structures can be digitally removed and anatomic relationships and orientations be viewed and investigated, which were previously obscured. This has obvious implications for research and educational applications. However, additional advantages may also be gained from use of these technologies, as a means to pre-operatively comprehend the natural drainage pathway and to formulate an approach tailored to maximize a patient's surgical outcome, before actually removing anatomic structures to gain access and visibility! Furthermore, the virtual surgery application may be used to plan and virtually execute the surgery and minimize the extent of structural removal, furthering the minimally invasive concept (Figure 1) [7]. 
We have seen, the field of endoscopic sinus surgery continue to evolve over the past 70 years in parallel with technological advances. As our understanding of paranasal sinus anatomy continues to improve, surgical techniques may be developed or refined, which adapt to the anatomic drainage system and adhere more completely to Kennedy's concept of restoring function, while preserving anatomic structures that do not require manipulation. The improvement in our understanding is best illustrated by what we thought about the Agger Nasi Cell [9], based on a 1988 direct sagittal CT and anatomic dissection of one specific specimen, (Figure 1: a-c) in which the ANC can be seen 'anterior' to the uncinate process, extending into the frontal recess and almost obstructing the 'frontal sinus ostium'. In retrospect, the Agger Nasi Cell illustrated in the sagittal CT of (Figure 1c) matches our new understanding of the Ethmoidal Uncinate Process in the image of (Figure 2c).
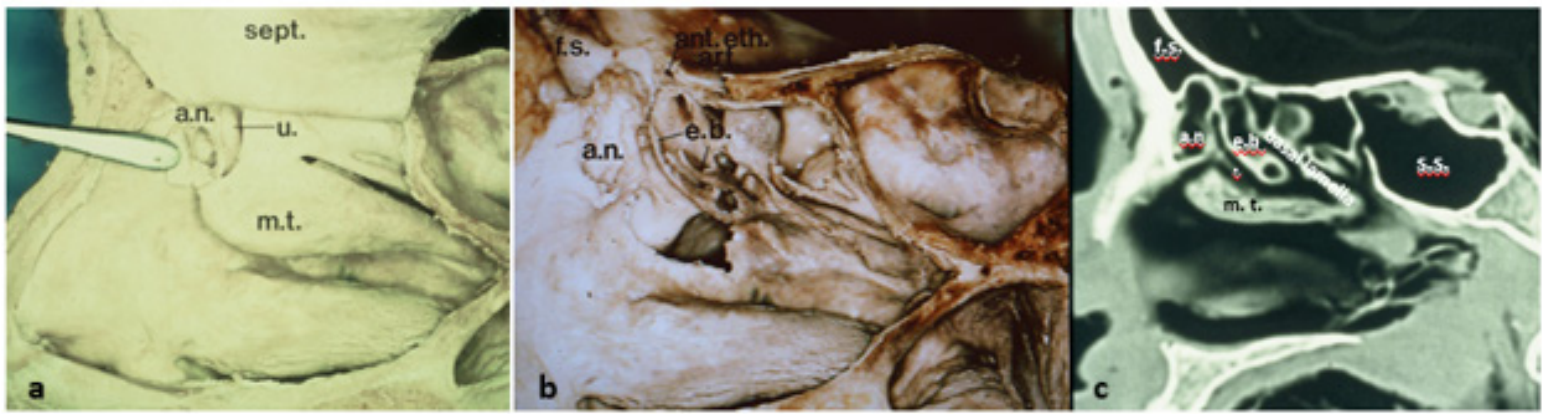

Figure 1: Previously available investigational methods, to evaluate sinus anatomy.

Figure ( $a, b)$ A dissected cadaver specimen status post partial resection of the nasal septum (sept.) as it is superiorly elevated figure (a), and removal of the medial surface of the lateral nasal wall figure (b), reveal the anterior lateral nasal wall anatomy: Agger Nasi (a.n.); middle turbinate (m.t.); Uncinate Process (u.); Frontal Sinus (f.s.); Ethmoid Bulla (e.b.); Sphenoid Sinus (s.s.); and Anterior Ethmoid Artery (ant.eth. art.).
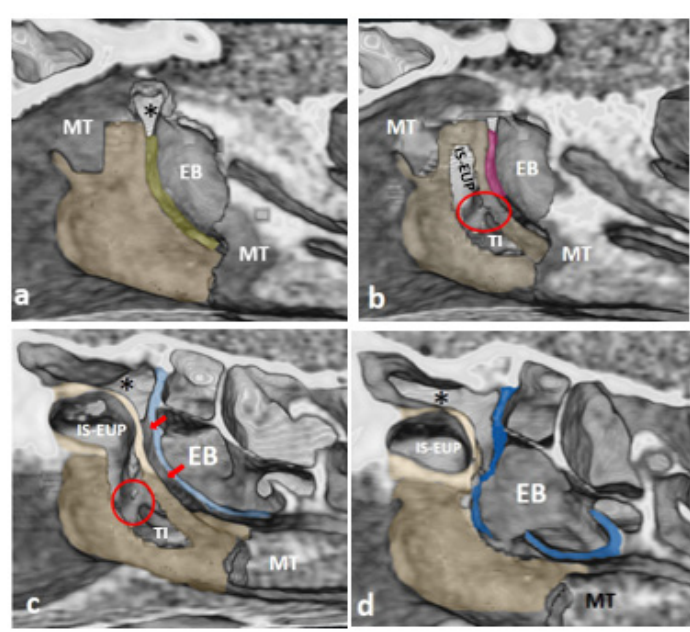

Figure 2: Recently available 3D CT Stereoscopic Imaging.

Figures (a-d) are 3D images revealing the anatomy of the lateral nasal wall status post partial removal of the middle turbinate (MT), with figure (a) being the most medial view and figure (d) being the most lateral view. Figure (a) reveals the relationship between the Uncinate Process (pale gold) and the ethmoid bulla (EB), as well as the relationship between the hiatus semilunaris (pale green) and the frontal recess $\left({ }^{*}\right)$. Figures $(b, c)$ show a partial virtual resection into the posterior wall of the Uncinate Process (pale gold), affording access to the Infundibular space within the Superior Ethmoid Uncinate Process segment (IS-EUP), as well as the Inferior Turbinal Uncinate process segment, the Turbinal Infundibulum (TI). Note the location of the ethmoid Infundibulum, in figure (b) (pink outline), and in figure (c) (red arrows). Communication between IS-EUP, $\mathrm{TI}$, and ethmoid infundibulum (red oval); Bulla Lamella (blue outline). Figure (d) reveals partial virtual resection of the posterior outline of the Uncinate lamella, as well as a more extensive resection of the bulla lamella (blue outline) affording a more extensive communication between the frontal recess $\left(^{*}\right)$, the ethmoid infundibulum, the ethmoid bulla (EB), and the Turbinal Infundibulum.

The contrast between the information available then in (Figure 1) and now with 3-DCTSI in (Figure 2) is dramatic. Previously, we considered the spaces within the anterior ethmoid to be cells, and that the uncinate was just a thin crescent shaped bony structure. Now with 3DCTSI, we can define the Uncinate's two components and demonstrate that the Ethmoidal Uncinate Process (EUP) segment pneumatizes superior and anterior, where it adheres to the Frontal Process of the Maxilla. As it develops and attaches to the lamina papyracea laterally, it encloses a space we have referred to previously as the ANC. However, this structure is in fact, the space 
enveloped by the bone of the uncinate process, as it develops. It is an Infundibular Space within the Ethmoidal segment of the Uncinate Process (IS-EUP), which communicates with the other two parts of the Infundibulum.

In the course of considering how this technology may give rise to new treatment approaches, we must also consider other things we have learned over the last 35 years about sinus physiology and minimally invasive techniques, such as:

A. Chronic rhinosinusitis is comprised of different types of inflammation, such as Eosinophilic and Non-eosinophilic inflammation, as noted by Stammberger in his many discussions and presentations. This has been further refined more recently as endotypes by Bachert and others.

B. A simple polyp or edematous mucosa biopsy in the office, analysis of nasal secretions, or asking for a tissue eosinophil count on the surgical specimen may be an important addition to the treatment armamentarium and can help distinguish the type of inflammation.

C. This information and the radiologic extent of disease, dictate how extensive the surgical treatment needs to be. Now that we have promising results from biologics/monoclonal antibody treatment of Eosinophilic chronic rhinosinusitis (ECRS) available, it may even suggest that surgery is not indicated as a first line of treatment in some cases.

D. Less invasive surgical techniques, such as balloon sinuplasty, may be developed and applied in a more targeted way to the specific areas involved with anatomic obstruction, thus sparing unnecessary removal of normal structures for "access" or "completeness".

E. As Helical CT has minimal effect on cataract formation [10], pre-operative medical treatment can oftentimes be safely documented with post-treatment CT to determine its effectiveness in difficult cases, whose outcome may affect the patient's other medical conditions, such as bronchiectasis, can be safely re-scanned post-surgery to exclude persistence of disease (the most common cause of surgical failure).

F. We have also learned that the intra-sinus Nitric Oxide concentration is important to the maintenance of sinus health and that overly large sinus openings, such as in the maxillary sinus, may act to equalize the nitric oxide concentration within the middle meatus/nasal cavity, thereby potentially removing a natural barrier to infection.

The above considerations and 3-DCTSI may open new horizons in sinusitis treatment and be of particular benefit to the challenging and variable anatomy of the frontal sinus outflow system. However, additional largely unexplored areas such as the skull base and the impact of neoplastic processes in displacing critical neurovascular structures using 3DCTSI may yield additional new insights.

\section{Acknowledgement}

None.

\section{Conflict of Interest}

N. London holds stock in Navigen Pharmaceuticals and was a consultant for Cooltech, Inc., neither of which are related to the present article. The other authors do not declare any conflicts of interest.

\section{References}

1. Messerklinger W (1972) [Technics and possibilities of nasal endoscopy] 20: 133-135.

2. Stammberger H, Posawetz W (1990) Functional endoscopic sinus surgery. Concept, indications and results of the Messerklinger technique. Eur Arch Otorhinolaryngol 247(2): 63-76.

3. Kennedy DW, Zinreich SJ (1998) The functional endoscopic approach to inflammatory sinus disease: current perspective and technique modifications. Am J Rhinology 2: 89-96.

4. Kennedy DW (1985) Functional endoscopic sinus surgery Technique. Arch Otolaryngol 111: 643-649.

5. Kane KJ (2020) The early history and development of functional endoscopic sinus surgery. J Laryngol Otol 134: 8-13.

6. Zinreich SJ, Kennedy DW, Rosenbaum AE, BW Gayler, A J Kumar, et al. (1987) Paranasal sinuses: CT imaging requirements for endoscopic sinus surgery. Radiology (163): 769-775.

7. Zinreich SJ, Kuhn FA, London NR, Kennedy DW, Solaiyappan M, et al. (2020) 3D CT stereoscopic imaging: an improved anatomical understanding of the anterior ethmoid sinus and frontal sinus drainage pathway. Rhinology Online 3: 202-220.

8. Schmale IL, Vandelaar LJ, Luong AU, Citardi MJ, Yao WC (2020) ImageGuided Surgery and Intraoperative Imaging in Rhinology: Clinical Update and Current State of the Art. Ear Nose Throat J 145561320928202.

9. Kuhn Frederick A, Bolger Wm E, Tisdal (1991) The Agger Nasi Cell in Frontal Recess Obstruction: An Anatomic, Radiologic and Clinical Correlation. Operative Techniques in Otolaryngology-Head and Neck Surgery 2(4): 226-231

10. Sillers, Michael J, Kuhn, Frederick A, Vickery, Christopher L (1995) Radiation Exposure in Paranasal Sinus Imaging. Otolaryngol Head Neck Surg 112: 248-251. 